\title{
Status and the perspectives of the Jiangmen Underground Neutrino Observatory (JUNO)*
}

\author{
Lino Miramonti (on behalf of JUNO Collaboration) \\ Department of Physics, Milano University and INFN, Sezione di Milano, \\ via Celoria 16, Milano I-20133, Italy \\ lino.miramonti@mi.infn.it
}

Received 26 February 2020

Accepted 26 February 2020

Published 13 March 2020

\begin{abstract}
One of the remaining undetermined fundamental aspects in neutrino physics is the determination of the neutrino mass hierarchy, i.e. discriminating between the two possible orderings of the mass eigenvalues, known as Normal and Inverted Hierarchies. The Jiangmen Underground Neutrino Observatory (JUNO), a 20 kt Liquid Scintillator Detector currently under construction in the South of China, can determine the neutrino mass hierarchy and improve the precision of three oscillation parameters by one order of magnitude. Moreover, thanks to its large liquid scintillator mass, JUNO will also contribute to study neutrinos from non-reactor sources such as solar neutrinos, atmospheric neutrinos, geoneutrinos, supernova burst and diffuse supernova neutrinos. Furthermore, JUNO will also contribute to nucleon decay studies. In this work, I will describe the status and the perspectives of the JUNO experiment.
\end{abstract}

Keywords: Neutrino oscillation; mass hierarchy; reactors; JUNO detector.

PACS Nos.: 14.60.Pq, 29.40.Mc, 28.50.Hw, 13.15.+g

\section{Introduction}

In the mid-1950s, Frederick Reines and Clyde L. Cowan built an experiment ${ }^{1}$ in order to verify the existence of the neutrino proposed by Wolfgang Pauli, more than 26 years before, as a desperate remedy to save the conservation laws of energy and momentum. They made use of reactor as a powerful source of electron antineutrinos. Since then, nuclear power reactors have been successfully employed to investigate the intimate nature of these elusive particles.

\footnotetext{
* Based on the presentation at the 20th International Workshop on Next generation Nucleon Decay
} and Neutrino Detectors (NNN19). 


\section{Miramonti}

The first experiments built to study neutrino properties and to investigate about a possible neutrino oscillation, as suggested by Bruno Pontecorvo, date back in the mid 1980s. The distance between the reactor and the detector (i.e. the baseline) was on the order of few decades of meters; Bugey, located at two distances, $13.6 \mathrm{~m}$ and $18.3 \mathrm{~m}$ from the reactor core, Savannah river at distances of $18.2 \mathrm{~m}$ and $23.8 \mathrm{~m}$, the ILL-Grenoble experiment, the Gösgen experiment, where data were collected at three different distances $37.9 \mathrm{~m}, 45.9 \mathrm{~m}$ and $64.7 \mathrm{~m}$, and last but not least Rovno and Krasnoyarsk. All these experiments have a baseline within $100 \mathrm{~m}$.

Starting from 1990, the distance of the detectors from the reactor core was extended to distances of the order of about $700 \mathrm{~m}$ for the Palo Verde ${ }^{2}$ experiment in US to about $1000 \mathrm{~m}$ in the case of the $\mathrm{CHOOZ}^{3}$ experiment in France.

Because of either their limited sensitivity or their short distance from the reactor core, all these experiments were not able to find any evidence of neutrino oscillation. Nevertheless, they played an important role in putting stringent constraints on mass and mixing parameters, in particular, the mixing angle between the first and the third neutrino generation (i.e. $\theta_{13}$ ).

At the beginning of the new millennium, the gigantic neutrino reactor experiment KamLAND ${ }^{4}$ measured reactor neutrino oscillations at a medium baseline of about $180 \mathrm{~km}$ providing new constrains on the solar neutrino oscillation parameters.

In 2011, based on the experience acquired with the aforementioned experiments, the value of $\theta_{13}$ was finally measured and turned out to be larger than previously assumed. Observing neutrino oscillation at a baseline of the order of $1 \mathrm{~km}$ Daya Bay, ${ }^{5}$ Double $\mathrm{Chooz}^{6}$ and $\mathrm{RENO}^{7}$ were able to measure the last mixing angle bringing the neutrino physics into the precision era.

The future generation of reactor neutrino experiments use tens of kilotons of liquid scintillator with a baseline of the order of $50 \mathrm{~km}$ in order to improve the neutrino oscillation parameters and shed light on neutrino mass ordering. An Asian detector is in construction in China the JUNO detector. ${ }^{14}$

\section{Reactor Neutrino Flux and Spectra}

\subsection{Reactor neutrino flux}

The reactor neutrino flux can be inferred with the fission rate of the isotopes in the reactor core and the corresponding cumulative neutrino spectrum per fission. These rates are estimated with the core simulation and the measurements of the thermal power. Starting from the inversion of the measured $\beta$ spectra of fissioning, the neutrino spectrum per fission of each isotope could be derived with an uncertainty of $2-5 \%,{ }^{8,9}$ The neutrino spectrum can also be derived by summation of thousands of decay branches of the fission products from nuclear database with an uncertainty of $\sim 10 \%, 9,10$

In nuclear power reactors, ${ }^{235} \mathrm{U},{ }^{238} \mathrm{U},{ }^{239} \mathrm{Pu}$ and ${ }^{241} \mathrm{Pu}$ perform fission reaction after absorbing a neutron. The fission products, being rich in neutrons, are unstable and undergoes $\beta$-decays. In each $\beta$-decay, one $\bar{\nu}_{e}$ (anti-electron neutrino) 
is produced. The typical energy of the neutrinos coming out from a reactor core corresponds to a $\beta$-decay energy of a few $\mathrm{MeV}$. About $6 \bar{\nu}_{e}$ 's are produced per fission releasing about $200 \mathrm{MeV}$, resulting in about $6 \cdot 10^{20} \bar{\nu}_{e}$ 's production per second for a $3 \mathrm{GW}_{\text {th }}$ thermal power reactor.

It is commonly used to refer to fission fractions in the core simulation instead of the fission rates. Fission fractions $\left(f_{i}\right)$ are defined as the ratio of the fission rate of an isotope over the total rate. The reactor neutrino spectrum can be calculated as follows:

$$
\Phi\left(E_{\nu}\right)=\frac{W_{\mathrm{th}}}{\sum_{i} f_{i} e_{i}} \cdot \sum_{i} f_{i} \cdot S_{i}\left(E_{\nu}\right),
$$

where $E_{\nu}$ is the neutrino energy and $e_{i}$ is the thermal energy released in each fission. $S_{i}\left(E_{\nu}\right)$ is the neutrino spectrum per fission for the $i$ th isotope. The thermal power $W_{\text {th }}$ can be measured to a sub-percent level.

\subsection{Spectra}

An electron anti-neutrino which interacts with a free proton in the target produces a positron and a neutron via inverse $\beta$-decay (IBD):

$$
\bar{\nu}_{e}+p \rightarrow e^{+}+n
$$

The positron produces a prompt signal carrying the energy information of the impinging neutrino and the neutron forms a delayed signal after thermalization; once captured, by a proton, it gives a strong signature of the event. The signature can be enhanced by adding nuclei with a high neutron capture cross-section such as gadolinium. The energy threshold of the inverse $\beta$-decay interaction is $1.8 \mathrm{MeV}$ and

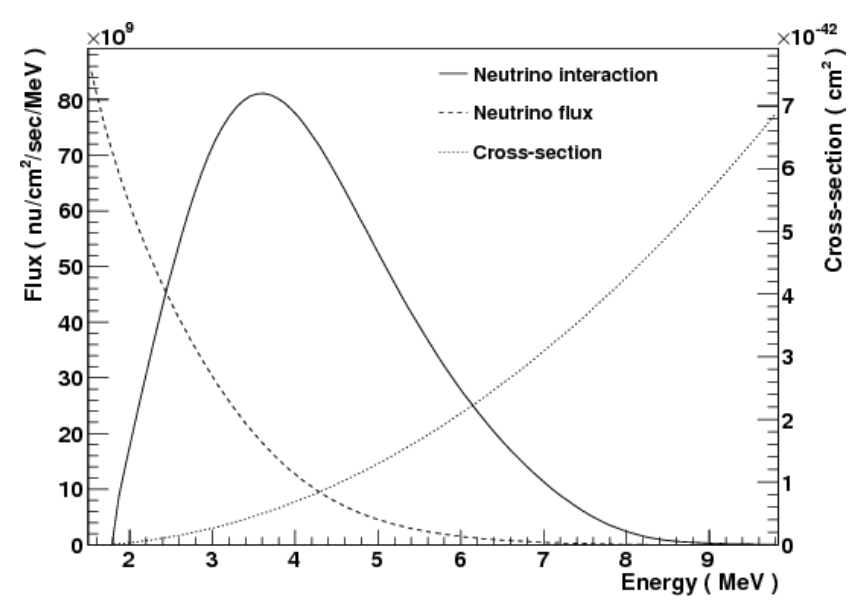

Fig. 1. Shape of neutrino energy spectrum in arbitrary units via inverse $\beta$-decay reactions expected in the detector (solid line). Overlaid curves show the reactor neutrino flux (dashed line) and cross-section of $\beta$-decay reaction (dotted line). Figure taken from Ref. 12 . 


\section{Miramonti}

the energy of the prompt signal is $E_{\text {signal }}=E_{\nu}-1.8 \mathrm{MeV}+2 m_{e} c^{2}$. Neutrons from inverse $\beta$-decay reactions are captured on hydrogen or by the gadolinium/boron nuclei and after thermalization $\gamma$-rays are emitted.

Figure 1 shows the reactor neutrino flux detected at the experimental site, the cross-section of inverse $\beta$-decay reaction and the shape of the energy spectrum in the detector are also shown. The number of interactions is obtained as a multiplication of the flux, interaction cross-section and the number of free protons in the detector according to Eq. (3).

The cross-section of inverse $\beta$-decay reaction is associated with the lifetime of free neutrons and could be calculated with an accuracy of $0.2 \% . \underline{11}$

$$
S\left(E_{\nu}\right) \simeq c \cdot \sum_{i} f_{i} \cdot S_{i}\left(E_{\nu}\right) \cdot \sigma\left(E_{\nu}\right)
$$

being $\sigma\left(E_{\nu}\right)$ the neutrino cross-section of inverse the $\beta$-decay reaction.

\section{The Neutrino Mass Hierarchy Determination}

Among the different open questions in neutrino physics, one of the most important concerns is the mass hierarchy of the tree neutrino mass eigenvalues. We know, from solar oscillation matter effects, that, indicating with $m_{1}$ the mass associated with $\nu_{1}$ and $m_{2}$ the mass associated with $\nu_{2}, m_{2}$ is larger than $m_{1}\left(m_{2}>m_{1}\right)$ but we do not know whether the value of $m_{3}$ associated to $\nu_{3}$ is larger or smaller than $m_{1}$. In the first case, we denote the ordering as "normal ordering" (NO) or "normal hierarchy" (NH): (i.e.: $m_{3} \gg m_{2}>m_{1}$ ), but we can also have the socalled "inverted ordering" (IO) or "inverted hierarchy" (IH): (i.e.: $m_{3} \ll m_{1}<m_{2}$ ), see Fig. 2.

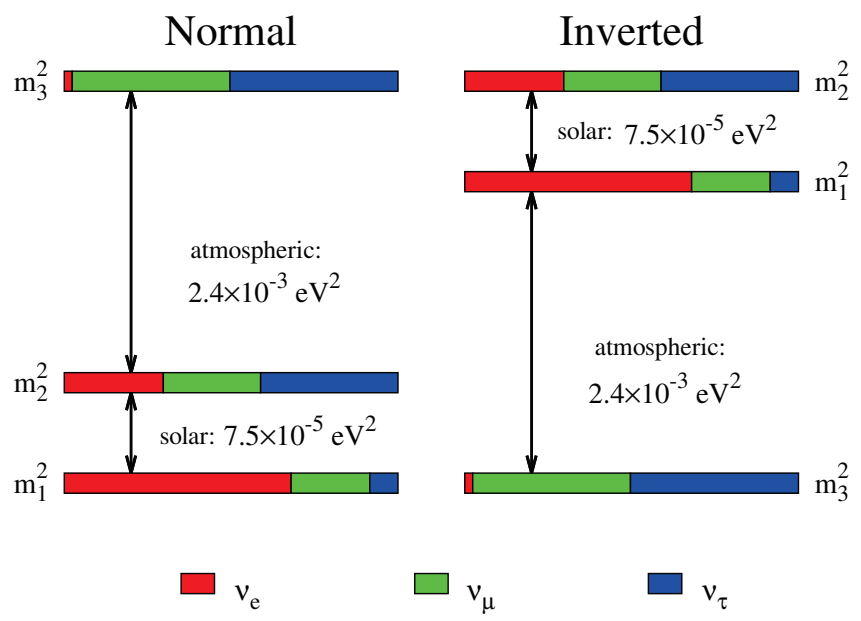

Fig. 2. Neutrino mass eigenstate flavor composition and mass pattern in the cases of normal and inverted hierarchies. 
In the three-flavor analysis framework, the $\bar{\nu}_{e}$ survival probability in vacuum is:

$$
\begin{aligned}
P_{e e}= & 1-\cos ^{4}\left(\theta_{13}\right) \sin ^{2}\left(2 \theta_{12}\right) \sin ^{2}\left(\Delta_{21}\right)-\sin ^{2}\left(2 \theta_{13}\right) \\
& \cdot\left[\cos ^{2}\left(\theta_{12}\right) \sin ^{2}\left(\Delta_{31}\right)+\sin ^{2}\left(\theta_{12}\right) \sin ^{2}\left(\Delta_{32}\right)\right]
\end{aligned}
$$

where

$$
\Delta_{i j}=\frac{\Delta m_{i j}^{2} L}{4 E}=\frac{\left(m_{i}^{2}-m_{j}^{2}\right) L}{4 E},
$$

$L$ (baseline) and $E$ (anti-neutrino energy) being the experimental parameters and $m_{i}$ and $m_{j}$ the neutrino mass eigenvalues.

The oscillation probability of Eq. (4) can be rewritten in the following way 13 :

$$
\begin{aligned}
P_{e e}= & 1-\cos ^{4}\left(\theta_{13}\right) \sin ^{2}\left(2 \theta_{12}\right) \sin ^{2}\left(\Delta_{21}\right)-\frac{1}{2} \sin ^{2}\left(2 \theta_{13}\right) \\
& \cdot\left[1-\sqrt{1-\sin ^{2}\left(2 \theta_{12}\right) \sin ^{2}\left(\Delta_{21}\right)} \cos \left(2\left|\Delta m_{e e}^{2}\right| \pm \phi\right)\right],
\end{aligned}
$$

where $\Delta m_{e e}^{2}$ represents the quantity $\Delta m_{e e}^{2}=\left[\cos ^{2}\left(\theta_{12}\right) \Delta m_{31}^{2}+\sin ^{2}\left(\theta_{12}\right) \Delta m_{32}^{2}\right]$ and the phase factor $\phi$ is the combination of the 1-2 sector mass and mixing parameters defined as:

$$
\begin{aligned}
\sin \phi & =\frac{\cos ^{2}\left(\theta_{12}\right) \sin \left[2 \sin ^{2}\left(\theta_{12}\right) \Delta_{21}\right]-\sin ^{2}\left(\theta_{12}\right) \sin \left[2 \cos ^{2}\left(\theta_{12}\right) \Delta_{21}\right]}{\sqrt{1-\sin ^{2}\left(2 \theta_{12}\right) \sin ^{2} \Delta_{21}}}, \\
\cos \phi & =\frac{\cos ^{2}\left(\theta_{12}\right) \cos \left[2 \sin ^{2}\left(\theta_{12}\right) \Delta_{21}\right]+\sin ^{2} \theta_{12} \cos \left[2 \cos ^{2}\left(\theta_{12}\right) \Delta_{21}\right]}{\sqrt{1-\sin ^{2}\left(2 \theta_{12}\right) \sin ^{2} \Delta_{21}}} .
\end{aligned}
$$

In the case of normal mass ordering, the sign in front of this term is equal to +1 while in case inverted mass ordering is equal to -1 .

In Fig. 3 is presented the convolution of the oscillation probability with the reactor anti-neutrino flux and the cross-section. The blue curve is obtained in the case of normal ordering while the red curve is in the case of inverted ordering. Thanks to the different pattern with a high statistics and good energy resolution and energy scale, it is possible to discriminate between the two possible hypothesis for the neutrino mass ordering.

To determine the neutrino mass ordering, a gigantic liquid scintillator detector is under construction near Jiangmen in the southern Chine at about $50 \mathrm{~km}$ from two nuclear power plants: the Jiangmen Underground Neutrino Observatory (JUNO). Beside the neutrino mass ordering determination, JUNO will measure some neutrino oscillation parameters (i.e. $\sin ^{2} \theta_{12}, \Delta m_{21}^{2}$ and $\left|\Delta m_{31}^{2}\right|$ ) with a precision better than $1 \%$ and foresees a rich program in astroparticle physics such as solar and atmospheric neutrinos detection, supernovae and diffuse supernova background, geoneutrino and proton decay searches. 


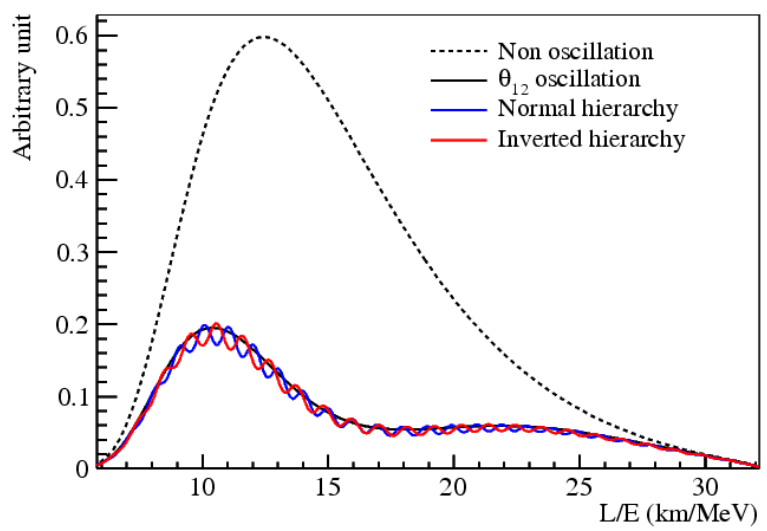

Fig. 3. (Color online) Reactor electron anti-neutrino spectrum as a function of L/E, in absence of oscillation and in the oscillation case for normal hierarchy (blue) and inverted hierarchy (red). Taken from Ref. 14, where it is reprinted with permission from Ref. 15.

\section{The JUNO Detector}

The JUNO detector was proposed in 2008 in the location site of Jiangmen city, Guangdong province, which is optimized to have the best sensitivity for mass hierarchy determination at a distance of about $53 \mathrm{~km}$ from both Yangjiang and Taishan nuclear power plants (NPPs), which the total thermal power is expected to be $26.6 \mathrm{GW}_{\text {th }}$ thermal power at the end of 2020. The detector is hosted in an underground laboratory under the Dashi hill overburden by $700 \mathrm{~m}$ of rocks. The experimental hall will have two accesses; a vertical shaft $563 \mathrm{~m}$ deep and a $1265 \mathrm{~m}$ long tunnel with a slope of $42 \%$. The expected number of IBD interactions collected via inverse $\beta$-decay is estimated to be on the order of 80 per day.

\subsection{The JUNO Central Detector}

The JUNO Central Detector consists in about 20 kton of active mass of Linear Alkyl Benzene (LAB) scintillator contained in an acrylic sphere of $35.4 \mathrm{~m}$ in diameter. The acrylic sphere is composed by 265 pieces of spherical panels with a thickness of $12 \mathrm{~cm}$ for a total net weight of about 600 tons. The liquid scintillator is doped with $3 \mathrm{~g} / \mathrm{L} 2.5$-diphenyloxazole (PPO) as fluor and $15 \mathrm{mg} / \mathrm{L} \mathrm{p}$-bis-(0-methylstyryl)benzene (bis-MSB) as wavelength shifter.

The LAB will be filtered with $\mathrm{Al}_{2} \mathrm{O}_{3}$ column to remove all impurities, beside a system handling distillation, water extraction and steam stripping of the LAB will be performed in order to reach the radiopurity requirements, the design goals are ${ }^{238} \mathrm{U}<10^{-15} \mathrm{~g} / \mathrm{g},{ }^{232} \mathrm{Th}<10^{-15} \mathrm{~g} / \mathrm{g}$ and ${ }^{40} \mathrm{~K}<10^{-17} \mathrm{~g} / \mathrm{g}$, respectively.

The light yield is about 1200 photoelectrons per $\mathrm{MeV}$. With a photocathode coverage larger than $75 \%$ and a PMTs PDE (photon detection efficiency) larger than $28 \%$ the detector will reach an unprecedented energy resolution of $3 \%$ at $1 \mathrm{MeV}$. 
The acrylic sphere is enclosed in a water pool with a depth of $44 \mathrm{~m}$ and a width of $43.5 \mathrm{~m}$ that will be used as Cherenkov veto and as shield for the external environment radiation and is lined with high density polyethylene plate (HDPE) in order to prevent the radon emanated from the rock to diffuse into the water. To increase the light collection, the surface of the detector is covered by Tyvek as reflecting and diffusing sheets. On top of the water pool, a muon tracker made of plastic scintillator modules to accurately measure the muon tracks has been placed.

\subsection{The JUNO PMT systems}

The light created inside the Central Detector is recorded by two complementary systems of PMTs; a set of large PMTs (LPMTs) and a set of small PMTs (SPMTs). The LPMT system is composed by about 18,000 PMTs of 20 inches in diameter, while the SPMT system consists in 25,000 PMTs of 3 inches in diameter. The LPMT system provides a coverage of about $75 \%$, while the SPMT system a coverage is about $3 \%$ for a total coverage larger than $78 \%$.

The LPMT system will have a dynamical range from 1 p.e. up to 1000 p.e., therefore they could be affected by nonlinearity effects whilst the range of the counting regime of the SPMT system will be in within 1-0 p.e., these last are also characterized by a better single p.e. resolution, a faster signal and a lower dark noise. The project of the front-end electronics is very challenging, due to a large number of read-out channels.

\subsection{The JUNO calibration systems}

If not properly calibrated, the non-uniformity would be one of the main effects contributing to spoil the liquid scintillator energy resolution. An efficient calibration system is of paramount importance in order to achieve an energy scale uncertainty better than $1 \%$. The calibration system will be composed of four complementary subsystems: an Automated Calibration Unit (ACU) which is a one-dimensional (1D) system and can be operated along the vertical axis, a Cable Loop System (CLS) which is a $2 \mathrm{D}$ system and can be operated to scan the vertical planes, a Guide Tube Calibration System (GT) which is a two a 2D system and can be operated to scan the outer surface of the CD, and a Remotely Operated Vehicle (ROV) which is a 3D system and can move freely within the CD to fully scan the whole detector. ${ }^{16}$ For more details see Ref. 17. For the calibration campaign, three kinds of radioactive source are foresees: radioactive $\gamma$-sources $\left({ }^{40} \mathrm{~K},{ }^{54} \mathrm{Mn},{ }^{60} \mathrm{Co},{ }^{137} \mathrm{Cs}\right), e^{+} / e^{-}$sources $\left({ }^{22} \mathrm{Na},{ }^{68} \mathrm{Ge},{ }^{40} \mathrm{~K},{ }^{90} \mathrm{Sr}\right)$ and $n$-sources $\left({ }^{241} \mathrm{Am}-\mathrm{Be},{ }^{241} \mathrm{Am}-{ }^{13} \mathrm{C},{ }^{241} \mathrm{Pu}^{13} \mathrm{C},{ }^{252} \mathrm{Cf}\right)$.

\section{The JUNO Physics program}

\subsection{The mass and mixing parameters determination}

Assuming an effective detection efficiency of $80 \%$ (considering event selection efficiency, live time and reactor full power) the number of inverse $\beta$-decay will be on the order of one hundred thousand in 6 years of data taking. 


\section{Miramonti}

In order to calculate the mass hierarchy sensitivity, we fit the simulated spectrum to the normal hierarchy $(\mathrm{NH})$ and inverted hierarchy $(\mathrm{IH})$ cases, respectively, using the $\chi^{2}$ test statistics.

$$
\chi^{2}=\sum_{i} \frac{\left(T_{i}-F_{i}\left(1+\epsilon+\epsilon_{i}\right)\right)^{2}}{T_{i}}+\sum_{p} \frac{\left(p_{f}-p_{t}\right)^{2}}{\sigma_{p}^{2}}+\sum_{i} \frac{\epsilon_{i}^{2}}{\sigma_{i}^{2}},
$$

where $T_{i}$ is the input anti-neutrino spectrum assuming the truth is normal hierarchy or inverted hierarchy, $F_{i}$ is the fitted spectrum, $\epsilon$ is the normalization factor of the anti-neutrino spectrum and it is floating in the $\chi^{2}$ test statistics. On $\epsilon$ there is any constrain, this means that we only use the shape information, and $\epsilon_{i}$ is uncorrelated between bins and so the shape can fluctuate.

The $p$ in $\chi^{2}$ test statistic denotes the oscillation parameters, including $\sin ^{2} 2 \theta_{13}$, $\sin ^{2} \theta_{12}, \Delta m_{21}^{2}$ and $\Delta m_{e e}^{2}{ }^{\text {a }}$

The $p_{f}$ and $p_{t}$ are fitted with the oscillation parameters and $\sigma_{p}^{2}$ is the uncertainty of the corresponding oscillation parameter.

Assuming the normal hierarchy is the truth (i.e. the corrected one), we try two fits using normal hierarchy spectrum and inverted hierarchy spectrum, respectively, and get two corresponding minimal $\chi^{2}$, that is $\chi_{\min }^{2}(\mathrm{NH})$ and $\chi_{\min }^{2}(\mathrm{IH})$. The discriminator can be defined as

$$
\Delta \chi^{2}=\chi_{\min }^{2}(\mathrm{IH})-\chi_{\min }^{2}(\mathrm{NH}) .
$$

With the nominal reactor power and 20 kton liquid scintillator detector with a $3 \% / \sqrt{E_{\text {vis }}}$ energy resolution and 6 years of data taking we obtain $\Delta \chi^{2} \simeq 11$ $(\simeq 3.3 \sigma)$.

The uncertainties associate to $\sin ^{2} 2 \theta_{13}, \sin ^{2} \theta_{12}, \Delta m_{21}^{2}$ have slight impacts to the sensitivity.

Beside the measurement of the neutrino mass hierarchy in 6 years of data taking, we will be able to determine $\theta_{12}, \Delta m_{21}^{2}$ and $\Delta m_{e e}^{2}$ with a precision of $0.67 \%, 0.59 \%$, $0.44 \%$, respectively, even if a more recent evaluations seem to suggest that the precision of these parameters will be slightly worse, but still below $1 \%$ for all them.

\subsection{Astroparticle physics program}

\subsubsection{Solar neutrinos}

Despite to be located at a depth of only $700 \mathrm{~m}$ below ground, JUNO will take advantage of it huge statistic and high-energy resolution. A key parameter for successfully measuring the neutrinos coming from the Sun is the low radioactivity level that the Collaboration has to achieve, in particular, for the analysis of ${ }^{7} \mathrm{Be}$ in the sub-MeV region.

A precise measurement of ${ }^{7} \mathrm{Be}$ and ${ }^{8} \mathrm{~B}$ fluxes could help in discriminating between the two different possible versions of the Standard Solar Model (low metallicity versus high metallicity) and to solve of the so-called "Solar Metallicity Problem".

a Effective mass-squared difference defined as a combination of $\Delta m_{31}^{2}$ and $\Delta m_{32}^{2} \cdot 13$ 


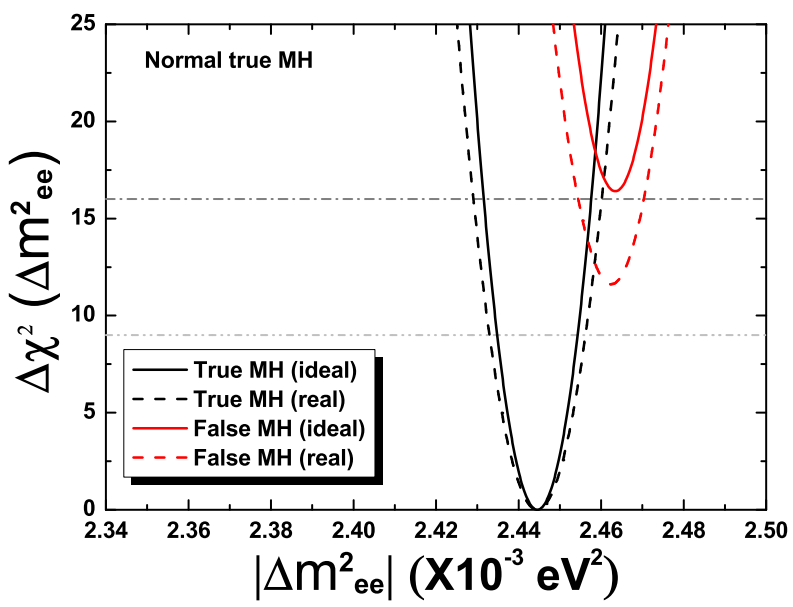

Fig. 4. Comparison of the MH sensitivity for the ideal and actual distributions of the reactor cores. Taken from Ref. 14 .

JUNO will be able to observe ${ }^{8} \mathrm{~B}$ neutrino events at "relatively low" energies allowing a detailed study of the spectrum in the transition energy region (around 3-4 MeV) of the anti-neutrino electron survival probability between the low-energy vacuum oscillation and the higher energies where the MSW mechanism effect dominates; thanks to that it will be possible to test the stability of the Large Mixing Angle (LMA) solar neutrino oscillation solution.

\subsubsection{Supernova neutrinos}

The main interaction channels that can be detected to study the neutrino emission by a Supernova are reported in Table 1, along with the number of expected events in JUNO for a Supernova at distance of $10 \mathrm{kpc}$.

About 5000 events for the main channel (i.e. the inverse $\beta$-decay) are expected and quite a significant number (of the order of hundreds of events) is also expected for the other interaction channels.

Table 1. Expected number of events in JUNO, for the main channels for neutrinos produced by a SN at a distance of $10 \mathrm{kpc}$. Taken from Ref. 14 .

\begin{tabular}{ccc}
\hline Process & Type & Events $\left(\left\langle E_{\nu}=14 \mathrm{MeV}\right)\right.$ \\
\hline $\bar{\nu}_{e}+p \rightarrow e^{+}+n$ & $\mathrm{CC}$ & $5.0 \times 10^{3}$ \\
$\nu+p \rightarrow \nu+p$ & $\mathrm{NC}$ & $1.2 \times 10^{3}$ \\
$\nu+e^{-} \rightarrow \nu+e^{-}$ & $\mathrm{ES}$ & $3.6 \times 10^{2}$ \\
$\nu+{ }^{12} \mathrm{C} \rightarrow \nu+{ }^{12} \mathrm{C}^{*}$ & $\mathrm{NC}$ & $3.2 \times 10^{2}$ \\
$\nu_{e}+{ }^{12} \mathrm{C} \rightarrow e^{-}+{ }^{12} \mathrm{~N}$ & $\mathrm{CC}$ & $0.9 \times 10^{2}$ \\
$\overline{\nu_{e}}+{ }^{12} \mathrm{C} \rightarrow e^{+}+{ }^{12} \mathrm{~B}$ & $\mathrm{CC}$ & $1.1 \times 10^{2}$ \\
\hline
\end{tabular}




\section{Miramonti}

\subsubsection{Geoneutrinos}

The pros of the JUNO detector in measuring neutrinos coming from the Earth interior are the radiopurity and the huge size. The cons are, of course, the presence of a very significant background, due to reactor anti-neutrinos which signal is similar to the one induced by geoneutrinos. ${ }^{18}$ However, we expect an improvement in geoneutrino measurement at JUNO site, which in 1 year of data taking should detect about $3-5$ hundreds of events. ${ }^{19}$

\section{Summary and Schedule}

In the South of China near Jiangmen city, the largest (20 kt target mass) and high precision ( $3 \%$ energy resolution at $1 \mathrm{MeV}$ ) liquid scintillator detector is under construction; the JUNO detector. This requirement is mandatory in order to determine the neutrino mass hierarchy through the detection of electron anti-neutrinos produced by two nuclear power plants with nominal power of $26.6 \mathrm{GW}_{\text {th }}$ thermal power at a baseline of about $53 \mathrm{~km}$. Besides the neutrino mass hierarchy determination JUNO aims to determine the neutrino mixing parameters $\theta_{12}, \Delta m_{21}^{2}$ and $\left|\Delta m_{31(32)}^{2}\right|$ with a precision better than $1 \%$. The JUNO Physics program extend beyond the reactor neutrino physics; it will drive its attention to neutrinos coming from supernova bursts, solar neutrinos and neutrinos originating from the inner layers of the Earth. The beginning of data taking is foreseen to begin in 2021 .

\section{References}

1. C. Cowan et al., Science 124, 103 (1956).

2. F. Boehm et al., Phys. Rev. Lett. 84, 3764 (2000).

3. M. Apollonio et al., Phys. Lett. B 466, 415 (1999).

4. K. Eguchi et al., Phys. Rev. Lett. 90, 021802 (2003).

5. F. P. An et al., Phys. Rev. Lett. 108, 171803 (2012).

6. Y. Abe et al., Phys. Rev. Lett. 108, 131801 (2012).

7. J. L. Ahn et al., Phys. Rev. Lett. 108, 191802 (2012).

8. P. Huber, Phys. Rev. C84, 024617 (2011) [Erratum-ibid. 85, 029901 (2012)].

9. A. C. Hayes et al., Phys. Rev. Lett. 112, 202501 (2014).

10. P. Vogel, G. K. Schenter, F. M. Mann and R. E. Schenter, Phys. Rev. C 24, 1543 (1981).

11. P. Vogel and J. F. Beacom, Phys. Rev. D 60, 053003 (1999).

12. H. Furuta et al., Nucl. Instrum. Meth. A 662, 90 (2012).

13. Y. F. Li, J. Cao, Y. Wang and L. Zhan, Phys. Rev. D 88, 013008 (2013).

14. F. An et al., J. Phys. G 43, 030401 (2016).

15. L. Zhan, Y. Wang, J. Cao and L. Wen, Phys. Rev. D 78, 111103 (2008).

16. Y. Zhang et al., J. Instrum. 14, P01009 (2019).

17. G. Zhu et al., Nucl. Sci. Tech. 30, 5 (2019).

18. M. Reguzzoni et al., J. Geophys. Res. Solid Earth 124, 4231 (2019).

19. R. Gao et al., Phys. Earth Planet. Inter. 299, 106409 (2020). 